\title{
Highly-Ordered Covalent Anchoring of Carbon Nanotubes on Electrode Surfaces by Diazonium Salt Reactions
}

\author{
Olimpia Arias de Fuentes, Tommaso Ferri,* Marco Frasconi,* Valerio Paolini, and \\ Roberto Santucci
}

Since their discovery in 1991, carbon nanotubes (CNTs) have attracted growing interest in view of their unique mechanical and physicochemical properties, ${ }^{[1,2]}$ and have successfully been applied in microelectronics ${ }^{[3-6]}$ such as memory devices, ${ }^{[4]}$ switches, ${ }^{[5]}$ supercapacitors, ${ }^{[6]}$ and in the biomedical area as drug-delivery devices ${ }^{[7]}$ and biosensors. ${ }^{[8-11]}$ The oriented assembly of CNTs on an electrode surface offers further possibilities with respect to both electrochemical biosensors because of the high electron-transfer rate along the tube, and the attachment of redoxactive molecules (or redox enzymes) to the ends of the CNTs. ${ }^{[10-14]}$

Electrodes modified with vertically aligned CNTs have been accomplished through direct growth ${ }^{[15,16]}$ or self-assembly ${ }^{[13,14,17,18]}$ of CNTs. Although different procedures can be followed to achieve a covalent attachment of molecules, ${ }^{[19]}$ the most applied method to anchor CNTs is perhaps that based on the formation of amide bonds from the reaction between the amines located on the modified electrode and the carboxylic groups at the ends and side-wall defects of the nanotubes.

Since 1992, when an aryl diazonium salt was used for the covalent functionalization of a carbon electrode ${ }^{[20]}$ this approach has been applied to a variety of surfaces ${ }^{[21]}$ such as carbon-based materials, ${ }^{[22-26]}$ metals, ${ }^{[27,28]}$ and semiconductors. ${ }^{[29]}$ The method has also been used for the derivatization of $\mathrm{CNTs}^{\left[{ }^{[3]}\right.}$ that is, multi-walled carbon nanotubes $(\mathrm{MWCNTs})^{[31]}$ or single-walled carbon nanotubes (SWCNTs). ${ }^{[30]}$

To the best of our knowledge, no report of CNT anchoring on a surface by the use of diazonium salt reactions has been published to date. The aim of the present work is to develop a new approach based on diazonium salt reactions that provides

[*] Prof. T. Ferri, V. Paolini

Department of Chemistry, "Sapienza" University of Rome

Piazzale Aldo Moro 5, 00185 Rome (Italy)

E-mail: tommaso.ferri@uniroma1.it

Dr. M. Frasconi

Department of Chemistry and Drug Technology

"Sapienza" University of Rome

Piazzale Aldo Moro 5, 00185 Rome (Italy)

E-mail: marco.frasconi@uniromal.it

Prof. O. Arias de Fuentes

Institute of Materials Science and Technology

University of Havana, Ciudad de La Habana (Cuba)

Prof. R. Santucci

Department of Medicine and Biochemical Science

"Tor Vergata" University of Rome (Italy)

믄

Supporting information for this article is available on the WWWW under http://dx.doi.org/10.1002/anie.201006743. a simple, stable, and well-organized assembly of CNTs for a wide range of substrates. To chemically modify an electrode surface, a diazonium salt is reduced to the corresponding aryl radical, which binds to the surface rapidly. ${ }^{[32-34]}$ The process is promoted by a variety of experimental conditions, ${ }^{[22]}$ and can even be used in combination with click chemistry. ${ }^{[27]}$

Herein, the procedures adopted for the derivatization of a glassy carbon electrode (GCE) are shown in Figure 1. For the immobilization of unfunctionalized SWCNTs on the chemically modified GCE surface, the p-nitro diazonium ions, which are formed in situ from $p$-nitroaniline (see Experimental Section for details), bind to the CGE surface (Figure 1a). The amine groups obtained by electrochemical reduction ${ }^{[35]}$ of nitro groups are converted into the corresponding diazonium functionalities, which covalently bind SWCNTs. The incorporation of phenyl groups to form a mixed monolayer, which is anticipated to prevent the diazo coupling, a reaction that occurs in a densely packed $p$-aniline monolayer. Indeed, the electrophilic attack of the diazonium functionality at the ortho position of a vicinal $p$-aniline (diazo coupling) or to the vicinal amine group, avoids CNTs immobilization. Alternatively, SWCNTs are first functionalized with $p$-nitrobenzene groups, then the amines formed by reduction of the nitro groups are converted into diazonium ions, which covalently bind SWCNTs on a clean GCE surface (Figure $1 \mathrm{~b}$ ).

The orientation of SWCNTs attached to a GCE surface was investigated by scanning electron microscopy (SEM). A typical SEM image, taken from a $45^{\circ}$ tilted view, is shown in Figure $1 \mathrm{c}$; the image clearly shows that SWCNTs are arranged vertically on the surface. The obtained arrangement is independent of the reaction route. TEM images clearly show that SWCNTs are perpendicularly oriented with only one end anchored on the CGE surface (Figure 1d,e). The SWCNTs attached to the GCE were $80-120 \mathrm{~nm}$ in length, with a diameter of $2-8 \mathrm{~nm}$.

Cyclic voltammograms (CVs) were recorded in a solution of $\mathrm{K}_{3}\left[\mathrm{Fe}(\mathrm{CN})_{6}\right]$ in water $\left(2 \times 10^{-3} \mathrm{~mol} \mathrm{~L}^{-1}\right)$ using bare GCEs or GCEs modified with SWCNTs according to the procedure described above (Figure 2). The assembly of CNTs on the electrode leads to a 1.5-fold increase of the current intensity, which results from the increased active electrode surface; the double-layer capacitance increases accordingly. Sonication of the modified electrode slightly decreases the current intensity, thus suggesting that some SWCNTs may be adsorbed on the surface. Also, the electrode modification slightly improves the reversibility, as indicated by the peak separation values. The electron-transfer rate constants $\left(k_{\mathrm{ET}}\right)$ determined for both bare and SWCNT-modified GCEs, were calculated from the peak separation assuming the $\left[\mathrm{Fe}(\mathrm{CN})_{6}\right]^{3-/ 4-}$ diffusion coef- 
a)

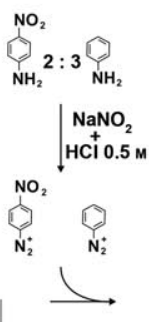

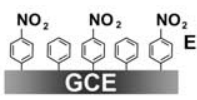

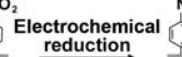

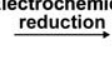

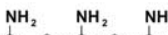

b)
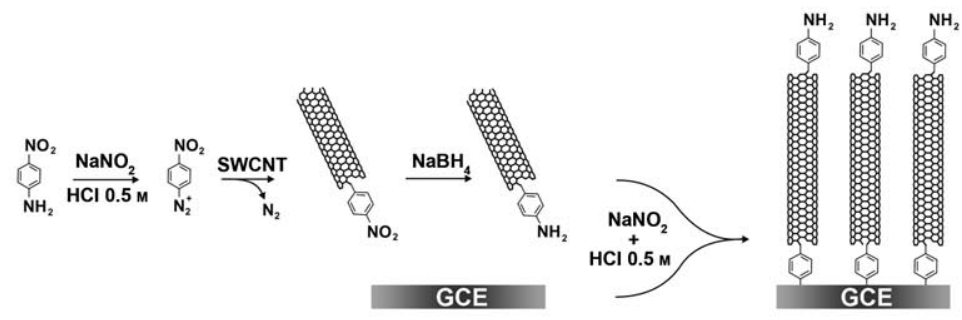

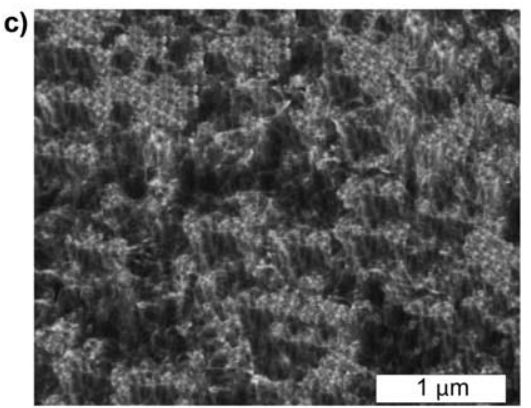

d)

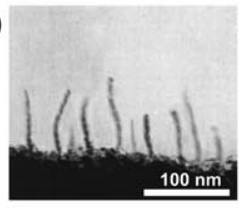

Figure 1. Schematic representation of SWCNT assembly on GCE. a) Immobilization of unfunctionalized SWCNTS on a GCE modified by a mixed monolayer of phenyl/aniline groups $3: 2$, obtained by the diazonium reaction. b) Self-assembly of aniline-functionalized SWCNTs on a bare GCE achieved by the diazonium reaction. c) SEM image of SWCNTs on a GCE (the SEM image was taken from $45^{\circ}$ tilted view). d),e) TEM images of SWCNTs on a GCE taken from different tilt angles.

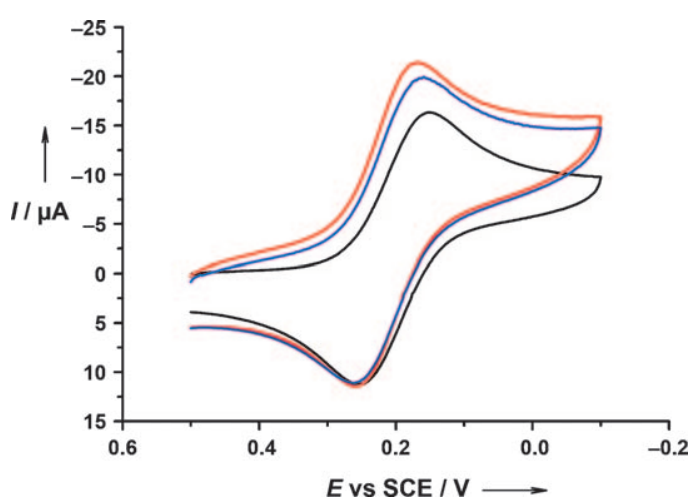

Figure 2. Cyclic voltammograms recorded at bare (black) and at a SWCNT-modified GCE (according to Figure $1 \mathrm{a}$ ), before (red) and after (blue) $10 \mathrm{~s}$ sonication. Measurements were carried out in $\mathrm{KCl}$ solution $\left(1 \mathrm{~mol} \mathrm{~L}^{-1}\right)$ that contained $\mathrm{K}_{3} \mathrm{Fe}(\mathrm{CN})_{6}\left(2 \times 10^{-3} \mathrm{~mol} \mathrm{~L}^{-1}\right)$; scan rate $v=50 \mathrm{mVs}^{-1}$.

ficient equals $5.7 \times 10^{-6} \mathrm{~cm}^{2} \mathrm{~s}^{-1}$. The value of $k_{\mathrm{ET}}$ determined at a bare GCE, $0.18 \mathrm{~cm} \mathrm{~s}^{-1}$, is consistent with the value reported in literature. ${ }^{[36]}$ The $k_{\mathrm{ET}}$ evaluated at a SWCNTmodified GCE before or after sonication is $0.13 \mathrm{~cm} \mathrm{~s}^{-1}$. As recently reported, SWCNTs vertically aligned on alkanthiol monolayers induce a decrease of $k_{\mathrm{ET}}$ by a factor of approximately $20,{ }^{[18]}$ which was ascribed to the presence of insulating tethered layers at the interface. In our measurements, the slight decrease of $k_{\mathrm{ET}}$ observed after electrode modification can be ascribed to defective sites present on the walls of the CNTs, which likely act as local barriers to electron transport. ${ }^{[13,18]}$ A similar electrochemical behavior was observed for diazonium-modified SWCNTs assembled on a bare GCE (see Figure S1 in the Supporting Information). In this case, the electrode modification was found to lead to a 1.5 -fold increase of the current intensity, which is consistent with an increased active surface; conversely, sonication lead to no effect on the electrochemical response. The $k_{\mathrm{ET}}$ derived, $0.12 \mathrm{~cm} \mathrm{~s}^{-1}$, implies that the free aryl amines located at the ends of SWCNTs have no effect on the electron-transfer parameters.

To shed more light on the versatility of this method, SWCNTs were anchored on the surface of a gold electrode through formation of a mixed self-assembled monolayer (SAM) ${ }^{[37,38]}$ of phenylthiol $/ p$-thioaniline $3: 2$, (Figure $3 \mathrm{a}$ ). In this case, the amino groups on the surface were converted into diazonium functionalities that are able to bind untreated SWCNTs suspended in solution. In an alternative approach, $p$-thioaniline was converted into the corresponding diazonium salt that is able to bind SWCNTs (Figure $3 \mathrm{~b}$ ). The $p$ thiophenyl-functionalized SWCNTs assembled on a bare Au electrode surface can form a self-assembled SWCNT monolayer. The AFM images of a gold electrode that was modified according to the described routes clearly show that SWCNTs are vertically aligned on the surface (Figure $3 \mathrm{c}, \mathrm{d}$ ). The orientation of the CNTs suggests that the diazonium reaction occurs mainly at the edges of the SWCNTs, which was also supported by UV/Vis/nIR and Raman spectroscopy (see Figure S2 and Figure S3 in the Supporting Information). Furthermore, sonication of the modified electrodes did not significantly change the density of the SWCNT assembly (see Figure S4 in the Supporting Information).

CVs recorded at a SWCNT-modified gold electrode show that sonication of the surface modified according to Figure $3 \mathrm{a}$ leads to a slight decrease in the recorded current intensity. Conversely, sonication of SWCNTs self-assembled on a gold surface results in no effect in the CVs (see Figure S5 in the Supporting Information). CVs recorded at a modified gold electrode do not show any significant differences from those recorded at a SWCNT-modified GCE, and the $k_{\mathrm{ET}}$ is $0.11 \mathrm{~cm} \mathrm{~s}^{-1}$ and $0.08 \mathrm{~cm} \mathrm{~s}^{-1}$ for bare and SWCNT-modified gold electrodes, respectively. The vertical arrangement of 


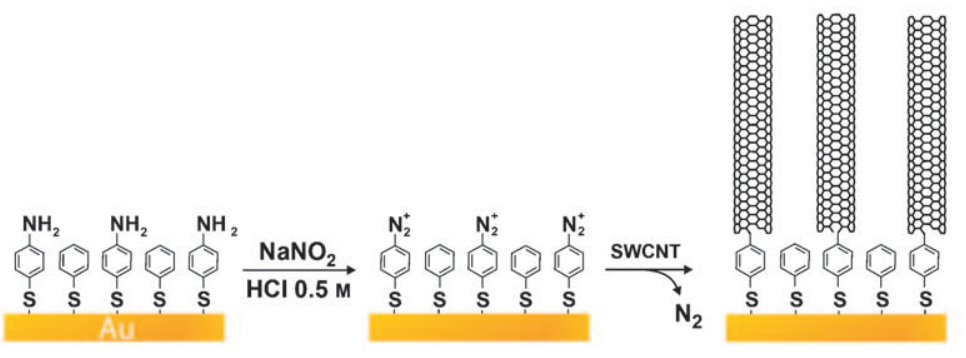

b)
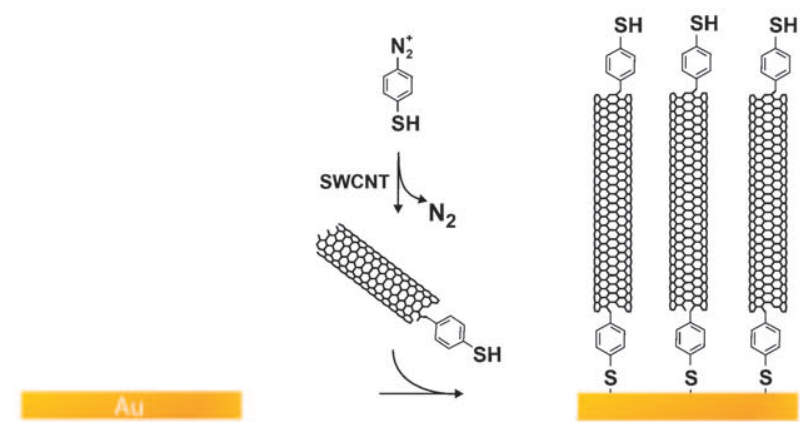

c)

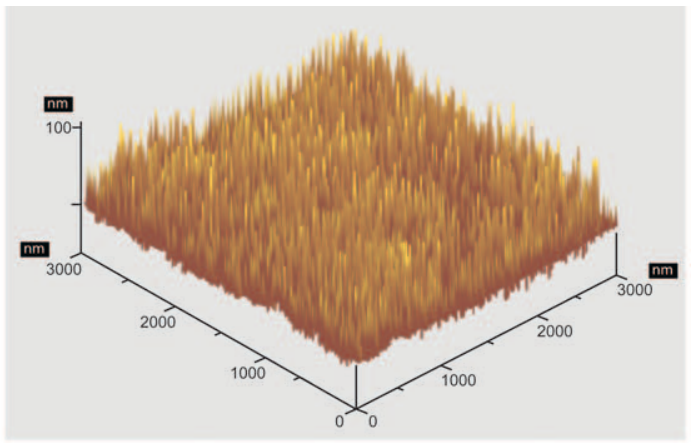

d)

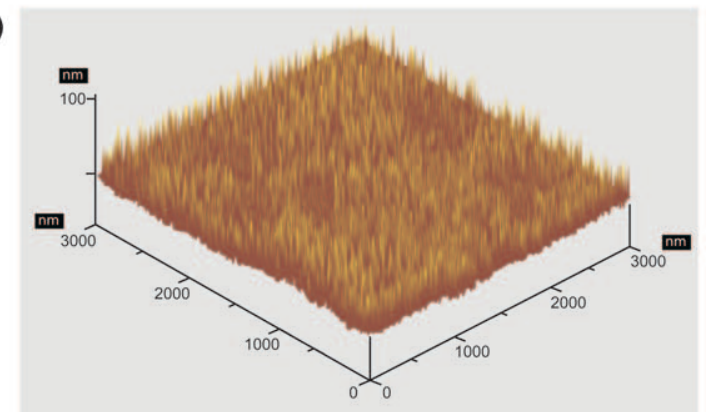

Figure 3. Schematic representation of SWCNT assembly on a gold electrode. a) Immobilization of unfunctionalized SWCNTs on a gold surface modified with a mixed SAM of thiophenol/p-aminothiophenol 3:2, obtained from a diazonium reaction. b) Self-assembly of thiophenol-

functionalized SWCNTs on a gold electrode. c), d) AFM images of an Au surface modified with SWCNTs according to the procedures described in (a) and (b), respectively.

SWCNTs, which is always observed on different electrode surfaces, can be better explained by the reactivity of diazonium salts towards SWCNTs, rather than the nature of the electrode surface: the ends of the SWCNTs are more reactive than the side walls. ${ }^{[39]}$ Further, the decrease of current intensity observed after sonication of a SWCNT-modified electrode (Figure 1a, Figure 3) suggests that some CNTs adsorb on the surface through $\pi-\pi$ interactions with the phenyl groups of the mixed SAMs; these SWCNTs can be easily removed by sonication. Conversely, no adsorption phenomenon was observed when the procedures shown in Figure $1 \mathrm{~b}$ and Figure $3 \mathrm{~b}$ were applied for electrode modification.

The assembly process of CNTs on a gold surface was investigated in situ by surface plasmon resonance (SPR) spectroscopy. A significant shift in plasmon angle as well as an increase in the minimum reflectance and broadening of the SPR curve was observed when SWCNTs were immobilized on a gold surface by following both routes described in Figure 3 (see Figure S6 in the Supporting Information). In a control experiment, the application of untreated SWCNTs on $p$-aminothiophenol/thiophenol SAMs before activation induced a very slight change in the SPR curve, hence suggesting that no binding of SWCNTs to the surface takes place. By comparing the sensorgrams for the two different cases, it is evident that the adsorption kinetics differ significantly (see Figure S7 in the Supporting Information): the assembly of untreated SWCNTs on aniline/phenyl SAMs occurs much more rapidly than that of thiol-functionalized SWCNTs. This observation can be ascribed to the different natures of the reactions that take place at the electrode surface (Figure $3 \mathrm{a}, \mathrm{b}$ ).

In order to develop biomaterial-based hybrid systems, we tested the compatibility of the SWCNT-modified electrodes with horse radish peroxidase (HRP), an enzyme that catalyzes the reduction of hydrogen peroxide. To this end, the enzyme was immobilized on a SWCNT-modified surface; the response of the HRP-SWCNT-modified electrode to increasing concentrations of hydrogen peroxide is illustrated in Figure 4. The typical electrocatalytic response of HRP was

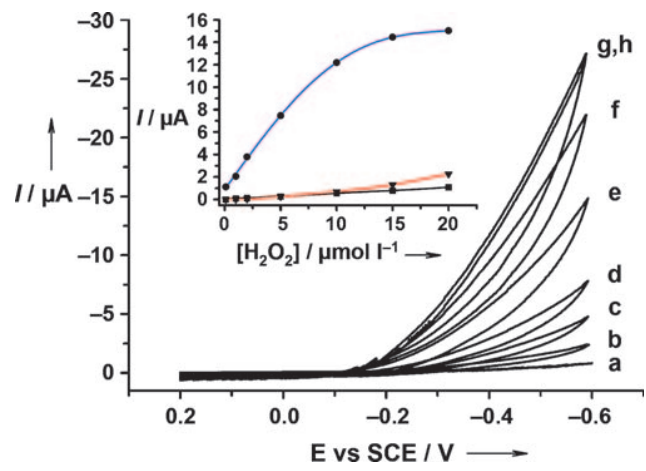

Figure 4. Cyclic voltammograms corresponding to the electrocatalytic reduction of $\mathrm{H}_{2} \mathrm{O}_{2}$ by a HRP-SWCNT-modified gold electrode; $\mathrm{c}\left(\mathrm{H}_{2} \mathrm{O}_{2}\right)$ in $\mu \mathrm{mol} \mathrm{L}^{-1}$ : a) 0.0 , b) 0.1 , c) 1.0 , d) 2.0 , e) 5.0, f) 10.0 , g) 15.0 , h) 20.0. The inset shows the calibration curve relative to the amperometric response (at $E=-0.45 \mathrm{~V}$ ) of a HRP-SWCNT- (blue), HRP-SAM- (red), and SWCNT- (black) modified gold electrode in the presence of different concentrations of $\mathrm{H}_{2} \mathrm{O}_{2}$. Measurements were carried out in TRIS buffer $(0.01 \mathrm{M}, \mathrm{pH} 7.0, I=0.1 \mathrm{M})$. Scan rate $v=10 \mathrm{mVs}^{-1}$. 
always detected at both GCE and SWCNT-modified gold electrodes. In a control experiment, the presence of $\mathrm{H}_{2} \mathrm{O}_{2}$ at a SWCNT-modified electrode, prior to HRP immobilization, gave a negligible signal (see Figure S8 in the Supporting Information). Furthermore, the immobilization of HRP on a SAM-modified electrode led to an electrocatalytic current for $\mathrm{H}_{2} \mathrm{O}_{2}$ reduction significantly lower than that observed in the presence of SWCNTs.

In conclusion, our study demonstrates that the covalent attachment of vertically aligned SWCNTs on a GCE or a gold surface, achieved by diazonium salt couplings, is possible and gives rise to an efficient system. The approach described herein is facile, versatile, and results in stable surfaces in consistency with the known stability of tether layers grafted from diazonium salts. It was shown that the assembly of CNTs on the tethered layer increases the electronic coupling between the electrode and the redox species in solution. Furthermore, this study highlights the suitability of SWCNTmodified electrodes for the successful development of biomaterial-based hybrid systems that are highly promising for future biotechnological applications.

\section{Experimental Section}

All electrochemical measurements were carried out by using a PAR model 273 potentiostat/galvanostat controlled by PAR model 270 electrochemical software (EG\&G Instruments, Princeton, NY, USA). GC and gold electrodes were used as working electrodes. All potentials were set against a SCE reference electrode; a Pt ring was used as the counter electrode. SPR experiments were carried out by using an ESPRIT instrument (Metrohm Autolab B.V., Utrecht, The Netherlands). AFM images were obtained with the help of a XE70 AFM (Park Systems) apparatus. A Philips XL30-FEG SEM operating at $10 \mathrm{kV}$, with the stage typically tilted at $45^{\circ}$, was used for SEM imaging. TEM images were obtained by using a Philips Tecnai 20 TEM unit. Visible and near-IR absorption data were collected on a Shimadzu UV-PC spectrophotometer model 3100. Raman spectra were measured by using both 633 and $785 \mathrm{~nm}$ excitation on a Renishaw Raman microscope model RM 2000 (Renishaw Inc., Hoffman Estates, IL).

SWCNTs were provided by Cheap Tubes Inc., USA. Throughout the experiments, reagents of analytical grade from Sigma and Carlo Erba and ultra pure water $\left(\rho \geq 18 \mathrm{M} \Omega \mathrm{cm}^{2}\right)$ from MilliQ Millipore system were used.

The procedure illustrated in Figure 1a was adopted as follows: a clean GCE was dipped in a $\mathrm{HCl}$ solution $\left(0.5 \mathrm{molL}^{-1}\right)$ containing $p$ nitroaniline $\left(4 \times 10^{-3} \mathrm{~mol} \mathrm{~L}^{-1}\right)$ and aniline $\left(6 \times 10^{-3} \mathrm{molL}^{-1}\right) . \mathrm{NaNO}_{2}$ $(10 \mathrm{mg})$ was added to the reaction mixture and left to react overnight. The modified GCE was sonicated in acetonitrile to eliminate all adsorbed molecules; then the nitro groups were electrochemically reduced to amine groups by scanning the potential from $-0.2 \mathrm{mV}$ to $-1.8 \mathrm{~V}$ at $100 \mathrm{mV} \mathrm{s}^{-1}$ in a $10 \%$ ethanolic solution $\left(\mathrm{KCl}=1 \mathrm{molL}^{-1}\right)$. The carefully rinsed electrode was immersed overnight in $1 \mathrm{~mL}$ of an SWCNT dispersion $\left(1 \mathrm{mg} \mathrm{mL}^{-1}\right)$ in $\mathrm{HCl}\left(0.5 \mathrm{molL}^{-1}\right)$ that contained $\mathrm{NaNO}_{2}(10 \mathrm{mg})$.

For the procedure illustrated in Figure $1 \mathrm{~b}$, CNT $(1 \mathrm{mg})$ was suspended in $\mathrm{HCl}\left(1 \mathrm{~mL}, 0.5 \mathrm{molL}^{-1}\right)$ and $p$-nitroaniline $(1 \times$ $\left.10^{-2} \mathrm{molL}^{-1}\right)$ and was sonicated for $4 \mathrm{~h} . \mathrm{NaNO}_{2}(10 \mathrm{mg})$ was then added and left to react overnight. The functionalized CNT suspension was centrifuged, the residue was washed with water and then centrifuged until the unreacted diazonium salt was completely removed (tested by $N$-(1-naphthyl)ethylenediamine). $p$-Nitrobenzene-modified SWCNTs were suspended in a basic solution $(\mathrm{pH} 10)$ and treated with $\mathrm{NaBH}_{4}$ as reducing agent; subsequently they were centrifuged and rinsed with water. The modified SWCNTs were suspended in $\mathrm{HCl}\left(0.5 \mathrm{molL}^{-1}\right)$ that contained $\mathrm{NaNO}_{2}(10 \mathrm{mg})$. The bare GCE was immersed in the suspension overnight.

The procedure illustrated in Figure $3 \mathrm{a}$ was adopted as follows: a clean gold electrode was dipped into an alcoholic solution of $p$ thioaniline $\left(4 \times 10^{-3} \mathrm{molL}^{-1}\right)$ and thiophenol $\left(6 \times 10^{-3} \mathrm{molL}^{-1}\right)$ to obtain a mixed SAM. SWCNTs were then anchored as reported for the procedure described in Figure $1 \mathrm{a}$.

For the procedure illustrated in Figure $3 \mathrm{~b}$, a naked gold electrode was immersed overnight in a suspension of $p$-thiophenyl-modified SWCNTs in ethanol.

For the HRP immobilization, HRP $\left(10 \mu \mathrm{L}, 1 \times 10^{-3} \mathrm{molL}^{-1}\right)$ in tris(hydroxymethyl)aminomethane (TRIS) solution $\left(0.1 \mathrm{~mol} \mathrm{~L}^{-1} \mathrm{KCl}\right.$ $1 \times 10^{-2} \mathrm{molL}^{-1}$ TRIS) were deposited onto the SWCNT-modified surface and left to dry in vacuum. The electrode was then rinsed with water.

Received: October 27, 2010

Revised: January 28, 2011

Published online: March 4, 2011

Keywords: biosensors - carbon nanotubes - diazonium salts . electron transfer $\cdot$ nanostructures

[1] S. Iijima, Nature 1991, 354, 56.

[2] V. N. Popov, Mater. Sci. Eng. R 2004, 43, 61.

[3] M. Bansal, R. Srivastava, C. Lal, M. N. Kamalasanan, L. S. Tanwar, Nanoscale 2009, 1, 317 .

[4] X. B. Lu, J. Y. Dai, Appl. Phys. Lett. 2006, 88, 113104.

[5] T. B. Martins, A. Fazzio, A. J. R. da Silva, Phys. Rev. B 2009, 79, 115413.

[6] Z. Fan, J. Yan, L. Zhi, Q. Zhang, T. Wei, J. Feng, M. Zhang, W. Qian, F. Wei, Adv. Mater. 2010, 22, 3723.

[7] K. Kostarelos, L. Lacerda, G. Pastorin, W. Wu, S. Wieckowski, J. Luangsivilay, S. Godefroy, D. Pantarotto, J. P. Briand, S. Muller, M. Prato, A. Bianco, Nat. Nanotechnol. 2007, 2, 108.

[8] E. Katz, I. Willner, ChemPhysChem 2004, 5, 1084.

[9] S. N. Kim, J. F. Rusling, F. Papadimitrakopoulos, Adv. Mater. 2007, 19, 3214.

[10] J. J. Gooding, Electrochim. Acta 2005, 50, 3049.

[11] A. Merkoci, M. Pumera, X. Llopis, B. Perez, M. del Valle, S. Alegret, Trac-Trends Anal. Chem. 2005, 24, 826.

[12] W. Yang, K. R. Ratinac, S. P. Ringer, P. Thordarson, J. J. Gooding, F. Braet, Angew. Chem. 2010, 122, 2160; Angew. Chem. Int. Ed. 2010, 49, 2114.

[13] F. Patolsky, Y. Weizmann, I. Willner, Angew. Chem. 2004, 116, 2165; Angew. Chem. Int. Ed. 2004, 43, 2113.

[14] J. J. Gooding, R. Wibowo, J. Liu, W. Yang, D. Losic, S. Orbons, F. J. Maarns, J. G. Shapter, D. B. Hibbert, J. Am. Chem. Soc. 2003, $125,9006$.

[15] Y. Yan, M. B. Chan-Park, Q. Zhang, Small 2007, 3, 24.

[16] B. Kim, H. Chung, W. Kim, J. Phys. Chem. C 2010, 114, 15223.

[17] X. Yu, B. Munge, V. Patel, G. Jensen, A. Bhirde, J. D. Gong, S. N. Kim, J. Gillespie, J. S. Gutkind, F. Papadimitrakopoulos, J. Rusling, J. Am. Chem. Soc. 2006, 128, 11199.

[18] A. Chou, P. K. Eggers, M. N. Paddon-Row, J. J. Gooding, J. Phys. Chem. C 2009, 113, 3203.

[19] M. Frasconi, F. Mazzei, T. Ferri, Anal. Bioanal. Chem. 2010, 398, 1545.

[20] M. Delamar, R. Hitmi, J. Pinson, J. M. Saveant, J. Am. Chem. Soc. 1992, 114, 5883.

[21] J. Pinson, F. Podvorica, Chem. Soc. Rev. 2005, 34, 429.

[22] J. M. Seinberg, M. Kullapere, U. Maeorg, F. C. Maschion, G. Maia, D. J. Schiffrin, K. Tammeveski, J. Electroanal. Chem. 2008, 624, 151. 
[23] K. Tammeveski, K. Kontturi, R. J. Nichols, R. J. Potter, D. J. Schiffrin, J. Electroanal. Chem. 2001, 515, 101.

[24] G. G. Wildgoose, M. Pandurangappa, N. S. Lawrence, L. Jiang, T. G. J. Jones, R. G. Compton, Talanta 2003, 60, 887

[25] G. Liu, M. Chockalingham, S. M. Khor, A. L. Gui, J. J. Gooding, Electroanalysis 2010, 22, 918.

[26] R. L. McCreery, Chem. Rev. 2008, 108, 2646.

[27] H. Gehan, L. Fillaud, N. Felidj, J. Aubard, P. Lang, M. M. Chehimi, C. Mangeney, Langmuir 2010, 26, 3975.

[28] J. L. Lyskawa, D. Baclanger, Chem. Mater. 2006, 18, 4755.

[29] B. S. Flavel, A. J. Gross, D. J. Garrett, V. Nock, A. J. Downard, ACS Appl. Mater. Interfaces 2010, 2, 1184.

[30] J. L. Bahr, J. P. Yang, D. V. Kosynkin, M. J. Bronikowski, R. E. Smalley, J. M. Tour, J. Am. Chem. Soc. 2001, 123, 6536.

[31] C. G. R. Heald, G. G. Wildgoose, L. Jiang, T. G. J. Jones, R. G. Compton, ChemPhysChem 2004, 5, 1794.

[32] F. Le Floch, J. P. Simonato, G. Bidan, Electrochim. Acta 2009, 54, 3078 .
[33] F. Anariba, S. H. DuVall, R. L. McCreery, Anal. Chem. 2003, 75, 3837.

[34] J. M. Noël, B. Sjoberg, R. Marsac, D. Zigah, J. F. Bergamini, A. F. Wang, S. Rigaut, P. Hapiot, C. Lagrost, Langmuir 2009, 25, 12742.

[35] P. Allongue, M. Delamar, B. Desbat, O. Fagebaume, R. Hitmi, J. Pinson, J. M. Saveant, J. Am. Chem. Soc. 1997, 119, 201.

[36] P. Chen, M. A. Fryling, R. L. McCreery, Anal. Chem. 1995, 67, 3115.

[37] J. C. Love, D. B. Wolfe, R. Haasch, M. L. Chabinyc, K. E. Paul, G. M. Whitesides, R. G. Nuzzo, J. Am. Chem. Soc. 2003, 125, 2597.

[38] J. C. Love, L. A. Estroff, J. K. Kriebel, R. G. Nuzzo, G. M. Whitesides, Chem. Rev. 2005, 105, 1103.

[39] C. E. Banks, A. Crossley, C. Salter, S. J. Wilkins, R. G. Compton, Angew. Chem. 2006, 118, 2595; Angew. Chem. Int. Ed. 2006, 45, 2533. 